\title{
Radiation Damage in Amplifiers Used for Quench Detection in a Superconducting Accelerator
}

\section{Remondino}

The behaviour of some isolation amplifiers irradiated at the CERN PSAIF facility is described. These components were irradiated to total integrated neutron fluences varying from $1.210^{13} \mathrm{n} / \mathrm{cm}^{2}(\mathrm{E}>140 \mathrm{keV})$ to $1.3410^{14} \mathrm{n} / \mathrm{cm}^{2}(\mathrm{E}>140 \mathrm{keV})$. The degradation of the electrical characteristics was measured versus doses. These amplifiers could be used for the superconducting magnet protection of LHC. They will be placed inside the tunnel and they will be exposed to $2.510^{13} \mathrm{n} / \mathrm{cm}^{2}$ ( $\mathrm{E}>140 \mathrm{keV}$ ) during 10 year operation life time.

Radec 95, Arcachon, France

Geneva, Switzerland

30 January 1996 


\title{
RADIATION DAMAGE IN AMPLIFIERS USED FOR QUENCH DETECTION IN A SUPERCONDUCTING ACCELERATOR
}

\author{
Vittorio Remondino
}

CERN, 1211 Geneva 23, Switzerland

\begin{abstract}
The behaviour of some isolation amplifiers irradiated at the CERN PSAIF facility is described. These components were irradiated to total integrated neutron fluences varying from $1.2 \times 10^{13} \mathrm{n} / \mathrm{cm}^{2}$ $(E>140 \mathrm{keV})$ to $1.34 \times 10^{14} \mathrm{n} / \mathrm{c} \mathrm{m}^{2}$ $(E>140 \mathrm{keV})$. The degradation of the electrical characteristics was measured versus doses. These amplifiers could be used for the superconducting magnet protection of LHC. They will be placed inside the tunnel and they will be exposed to $2.5 \times 10^{13} \mathrm{n} / \mathrm{cm}^{2}(\mathrm{E}>140 \mathrm{keV})$ during 10 year operation life time.
\end{abstract}

\section{INTRODUCTION}

CERN intends to build a particle accelerator (the Large Hadron Collider - LHC) using superconducting magnets. This machine will be installed in the tunnel of the existing Large Electron Positron (LEP) collider.

In the LHC many hundreds of large superconducting magnets will be powered in series to give the necessary bending power to maintain protons in their circular orbit. Such large superconducting magnets will run at the limit of existing technology in a high radiation environment.

Superconducting magnets are liable to loose their superconductivity or "quench" under fault conditions. The extremely high energy density of the LHC magnets could cause local overheating of the magnet in the section of the coil where the quench starts. To avoid this, the incipient quench must be detected as soon as possible. A signal must then be sent to fire strip heaters, which provoke quenching along the outer layer of the magnet coil. This spreads the energy out through a large volume of the winding, limiting the maximum temperature rise and maximum internal voltages [1]. Without the heaters the magnet could be destroyed by the local overheating.

The detection of quench is based on measurements of voltage between the ends of the two coils of each magnet. If a quench occurs, then a differential voltage appears and can be detected [2]. In order to trigger the protection system, these detection signals must be well above the noise level.

In addition to the quench detection amplifiers, a second set of amplifiers will continuously monitor the magnet voltages. When a fault occurs, the voltage wave form is recorded. This helps to localise and understand the origin of the quench and to identify "false" quenches $[1,2]$.
For transmitted signals the pick-up noise level is proportional to the length of the cable. Therefore, amplifiers which measure and amplify the voltages across the magnet must be situated in the tunnel near the magnet. Amplifiers must not only amplify the signals but also isolate the detection system from the magnet power circuit.

Electronic isolation amplifiers are clearly suitable for this requirements but they must be "radiation hard" to the levels given by LHC.

\section{IRRADIATION AND DOSIMETRY}

The dose in the LHC tunnel was estimated considering a proton beam of $8 \mathrm{TeV}$ and taking into account the continuous losses due to the beam halo hitting the collimators and the vacuum chamber, the beam losses due to beam scattering and the accidental losses of the primary proton beam [3]. These calculations are made assuming continuous losses on the magnets of $10^{6}$ protons per second, just below the alarm threshold of the beam loss monitoring system, for a year of operation (taken to be $10^{7}$ seconds).

The dose and neutron fluences are given in Fig. 1 at various radial positions, from the beam centre to the tunnel walls for the nominal beam parameters and for the large majority of the magnets [4]. The neutron

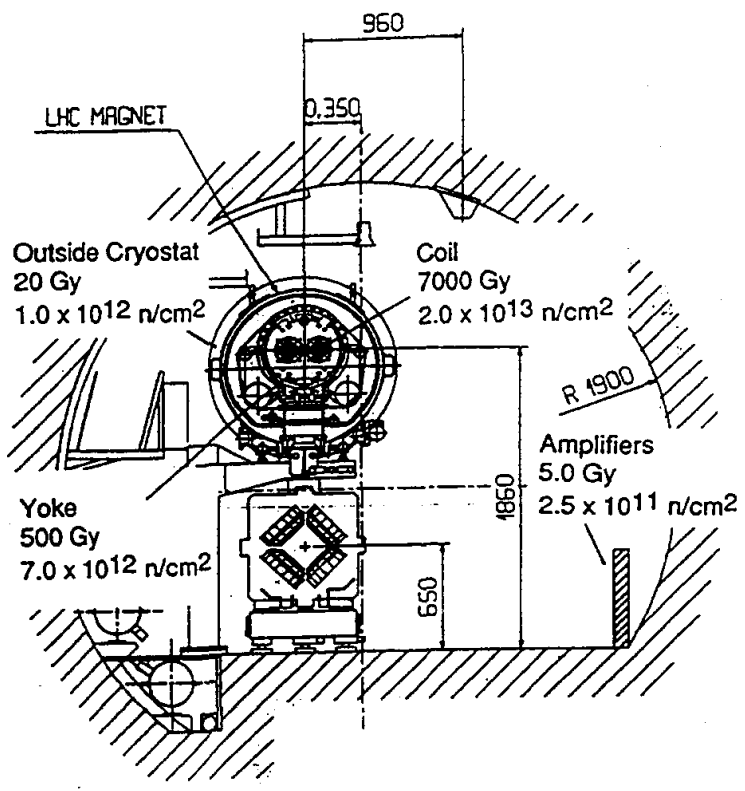

Fig 1. Doses to LHC dipoles per year 
fluences have been cut at $140 \mathrm{keV}$ since displacement damage in semiconductors is negligible below that energy.

Systematic measurements at the existing CERN machines have shown that dose fluctuations of a factor ten are typical for proton accelerators, therefore the dose and fluence are expected to be $50 \mathrm{~Gy}$ and $2.5 \times 10^{12} \mathrm{n} / \mathrm{cm}^{2}(\mathrm{E}>140 \mathrm{keV})$ per year at the tunnel wall where the amplifiers will be placed.

In order to investigate the influence of irradiation on the electrical characteristics of the amplifiers, the circuits have been submitted to preliminary radiation tests at the CERN PS-Acol Irradiation Facility (PSAIF). The irradiation area is sited behind the antiproton production target. During the production of antiprotons, this target is hit by a $26 \mathrm{GeV}$ protons beam from the Proton Synchrotron (PS). At the irradiation place, a typical accelerator radiation field is then produced by the strong emission of secondary high-energy particles and neutrons. A container (18 $\mathrm{cm}$ in diameter and $20 \mathrm{~cm}$ in height) can be lowered along a tube to variable distance from the accelerator beam. The distance from the beam yields a variable dose rate. On-line measurements can be carried out in a safe radiation environment during the irradiation.

As described in [5], the dose inside the container has been measured as a function of the number of protons on the target.

Radiophotoluminiscence (RPL) dosimeters, thermoluminescence dosimeters (TLD), polymer/alanine dosimeters (PAD) were used for dose measurements. Activation detectors and TLDs were used for neutron flux measurements and for evaluating the neutron spectrum by means of the LOUHI program [6]. The shape of the energy-spectrum of the neutrons, shown in Fig. 2, is given in $\mathrm{n} / \mathrm{cm}^{2}$. $s$ or in logarithmic energy bin Flux/BN/Log.

The values to be taken into account for the irradiation of our circuits are of $50 \mathrm{mGy}$ (total dose mainly gammas) per pulse of $1.4 \times 10^{13}$ protons and $1.1 \times 10^{9} \mathrm{n} / \mathrm{cm}^{2}$ (E>140 keV) per pulse. According to the shape of the spectrum, the neutron fluence (E $>140 \mathrm{keV}$ ) is almost equivalent to the fluence at a $1 \mathrm{MeV}$ equivalent dose. This was confirmed by measurements carried out with PIN diodes in 1993 [7].

TABLE 1

LIST OF ALL EXPOSURESON THE AMPLIFIERS

\begin{tabular}{|r|l|l|r|r|}
\hline $\begin{array}{l}\text { Exp. } \\
\text { Nr. }\end{array}$ & Components Nr & $\begin{array}{l}\text { Dose } \\
\text { meas. } \\
{[\mathrm{Gy}]}\end{array}$ & Dose rate & $\begin{array}{l}\text { Fluence } \\
{\left[\mathrm{n} / \mathrm{cm}^{2}\right]}\end{array}$ \\
\hline $\mathrm{Gy} /$ hour $]$ & $\mathrm{E}>140 \mathrm{KeV}$ \\
\hline 1 & $\mathrm{AD} 289, \mathrm{ISO} 106$, & 500 & 5 & $1.10 \times 10^{13}$ \\
& PWS727, DESY & & & \\
2 & AD289 (G=10) & 3400 & 25 & $7.60 \times 10^{13}$ \\
3 & AD289 (G=30,90) & 2000 & 25 & $4.50 \times 10^{13}$ \\
4 & AD210 (G=20) & 6000 & 25 & $1.34 \times 10^{14}$ \\
\hline
\end{tabular}

Table 1 shows the list of all radiation exposure levels and the dose rate on the amplifiers. The temperature during the irradiation was in the range between $18^{\circ}$ and $25^{\circ} \mathrm{C}$. With each set of components, some radiophotoluminiscence dosimeters (RPL) were irradiated. The number of protons on the target was monitored to evaluate the absorbed dose all along the exposures. The degradation of the performance of the amplifiers was then measured as a function of the dose. Finally, the final dose was compared to the RPL readings at the end of each exposure.

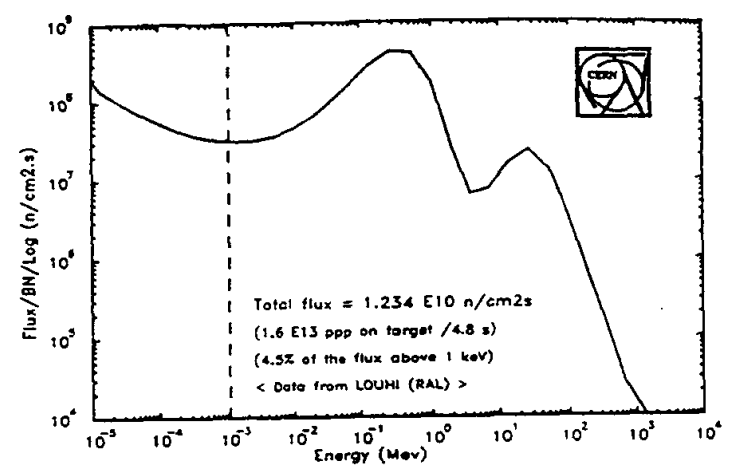

Fig. 2. Neutron Spectrum in the PSAIF container

\section{CHARACTERISTICS OF THE CIRCUITS TESTED}

The electrical characteristics of the amplifiers required for our purpose are not specially severe: the quench detection level is between 0.1 and $0.5 \mathrm{~V}$, the frequency response rates range from dc to a few $\mathrm{kHz}$, the gain between 10 and 50 , the voltage isolation between inputs and outputs is of the order of one thousand volts.

With a view to a complete isolation and protection from line transients, the following components have been chosen:

Isolation amplifiers: AD289J, AD210JN, ISO106 $\mathrm{dc} / \mathrm{dc}$ converters: PWS727

Magnetic amplifier : made by DESY (Deutsches Elektronen-Synchrotron lab in Hamburg, Germany) for HERA (High Energy Ring Accelerator)

\section{A. AD289J and AD210JN}

Such amplifiers are a wide band, accurate, lowcost isolation amplifiers made by ANALOG DEVICES. These circuits are manufactured with surface-mounted components. They provide a complete isolation via a transformer coupling internal to the module. They are powered by a single $+15 \mathrm{~V}$ supply, and $a \pm 15 \mathrm{~V}$ isolated power is internally generated and supplied to both the input and output ports. This eliminates the need for an external DC/DC converter. They provide high accuracy with complete 
galvanic insulation and protection from line transients or fault voltages.

The AD289J model offers a guaranteed gain nonlinearity error of $\pm 0.05 \%$, frequency response ( $-3 \mathrm{~dB}$ ) from dc to $20 \mathrm{kHz}$ and isolation between input and output of $\pm 2500 \mathrm{~V}$.

The AD210JN model assures a maximum nonlinearity of $\pm 0.025 \%$, a bandwidth of $20 \mathrm{kHz}$ $(-3 \mathrm{~dB})$ and isolation of $2500 \mathrm{~V}$ between any ports.

\section{B. ISO106}

This amplifier is a unity gain buffer isolation amplifier made by BURR BROWN intended for level input voltages on the order of $1 \mathrm{~V}$ to $10 \mathrm{~V}$. They may be preceded by amplifiers that precondition a low level signal and translate it to a high level. The amplifier isolates $\pm 10 \mathrm{~V}$ analogue signals by digitally encoding the input voltage and uniquely coupling across a differential ceramic capacitive barrier $(3 \mathrm{pF})$.

The continuous isolation is rated for $3500 \mathrm{~V} \mathrm{rms}$; the typical frequency response varies from dc to $70 \mathrm{kHz}$; the non linearity gain is $\pm 0.04 \%$ and the initial input offset voltage is in the range of $\pm 25 \mathrm{mV}$.

\section{C. $P W S 727$}

The PWS727 is an isolated, unregulated $\mathrm{dc} / \mathrm{dc}$ converter designed by BURR BROWN. It converts a single input voltage $(10 / 18 \mathrm{Vdc})$ to bipolar voltages of the same value as the input. It provides a minimum isolation voltage of $1500 \mathrm{~V} \mathrm{rms}$, output currents of $\pm 15 \mathrm{~mA}$ at voltage accuracy of $\pm 0.75 \mathrm{~V}$. This circuit could be used for powering an isolation amplifier without an integrated power supply, like the ISO106.

\section{DESY Magnetic Amplifier}

This is a low impedance passive device in which a current amplification is obtained by using non linear properties of ferromagnetic materials.

The ac circuit and the control circuit (Fig. 3) are magnetically coupled by means of a high permeability metal-glass core driven by a $200 \mathrm{~Hz}$ ac voltage [8]. When $E_{c}=0$, the total voltage $E_{a}$ applied to the ac circuit is shared between the resistive and the inductive charge. During the linear part of the magnetization curve, the inductance is high, the voltage drops across the resistance $R$ is then small and the same is true for the current $i_{a}$. If the applied voltage $\left(E_{a}\right)$ is such as to bring the circuit almost into the saturation zone the inductance falls. Most of voltage appears across the resistance $\mathrm{R}$ with a consequent high value for the current $\left(I_{2}\right)$. The circuit behaviour is then non linear.

In operational conditions, the supply voltage $E_{a}$ is adjusted so that its peak value just fails to saturate the magnetic core with $E_{c}=0$. If an input voltage, $\left(E_{c} \neq 0\right)$, is then applied to the control circuit, the corresponding current will change the flux in the core.
Also, as a consequence, the current $\left(\mathrm{I}_{\mathrm{a}}\right)$ and the voltage across the resistance $R$ will vary proportionally. The device is usually completed by ancillary equipment as a test winding (output 1-2 in Fig. 3). At the Hera machine a quench signal of $-100 \mathrm{mV}$ is applied to the control circuit: the resulting current in the ac circuit produces a $10 \mathrm{~V}$ signal on $\mathrm{R}$. The circuit behaviour is therefore strongly non linear: it can not be used as a monitoring amplifier (recording of data) but only as a threshold switch. It has been tested because it is expected to be radiation resistant. Moreover, it is cheap, reliable (no maintenance required), robust and has good voltage insolation. Its response in time is longer than $1 \mathrm{~ms}$ and its frequency response limited to $\sim 100 \mathrm{~Hz}$, and is thus suitable for our applications.

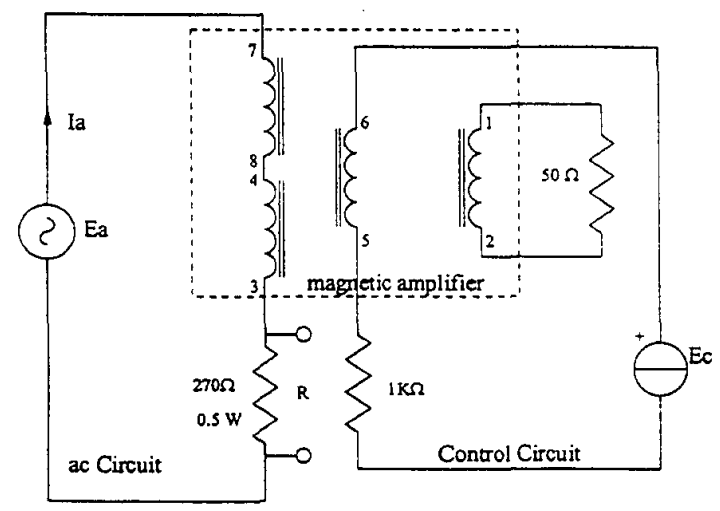

Fig. 3. Magnetic amplifier test circuit

\section{TESTING PROCEDURES}

Tests were carried out on completed electronic circuits, mounted on a printed board and placed in the container in the irradiation area. All instrumentation needed to test and power the circuits was located outside the irradiation facility in a safe environment.

The electrical characteristics of each component were measured prior to the irradiation. During the exposures, all measurements were made in situ to take data without stopping the irradiation.

Parameters monitored were: power consumption, dc gain $\left(\mathrm{V}_{\mathrm{i}}= \pm 5 \mathrm{~V}\right.$ for ISO106, $\mathrm{V}_{\mathrm{i}}= \pm 0.5 \mathrm{~V}$ for AD289, $A D 210$ ), ac gain at $100 \mathrm{~Hz}, 1 \mathrm{Khz}$ and $10 \mathrm{Khz} \quad\left(\mathrm{V}_{\mathrm{i}}=3 \mathrm{~V}_{\text {peak }}\right.$ for ISO106, $\mathrm{V}_{\mathrm{i}}=1 \mathrm{~V}_{\text {peak }}$ for $\mathrm{AD} 289, \mathrm{AD} 210)$ and the offset voltage. An isolation test $\left(1000 \mathrm{~V}_{\mathrm{rms}}\right.$ between input and output) was made at the beginning and at the end of the exposures on the ISO106, AD289 and AD210.

A batch of 2 samples of ISO106, PWS277, $\mathrm{AD} 289 \mathrm{~J}$ and one magnetic amplifier was subjected to the first radiation test. The total fluence of $1.1 \times 10^{13} \mathrm{n} / \mathrm{cm}^{2}(E>140 \mathrm{keV})$ was obtained over a period of about one month. At the beginning of the experiment, the dose level was kept low to monitor any possible initial degradation of electrical characteristics. Dose levels were then progressively increased 
and no appreciable changes were observed below a fluence of $2.9 \times 10^{12} \mathrm{n} / \mathrm{cm}^{2}(\mathrm{E}>140 \mathrm{keV})$.

The second exposure was performed to examine the behaviour of AD289J components in circuits with a gain of 10: the two samples previously irradiated were exposed to an additional fluence of $7.6 \times 10^{13} \mathrm{n} / \mathrm{cm}^{2}$ (E>140 keV) during 13 days. The characteristics of the two AD289Js were checked before the new exposure test. The components did not show any change in performance. This fact excludes any selfannealing effect. Eight new components were added to the test series.

The AD289J were tested again in a third irradiation test to examine their behaviour at different gains of 30 and 90 . Ten new amplifiers were assembled on printed circuit, in pairs, exactly as in the second test and exposed to a fluence of $4.5 \times 10^{13} \mathrm{n} / \mathrm{cm}^{2}$ $(\mathrm{E}>140 \mathrm{keV})$ in 7 days.

Finally, during the fourth exposure, a batch of ten AD210JN amplifiers was exposed to a fluence of $1.34 \times 10^{14} \mathrm{n} / \mathrm{cm}^{2}$ (E>140 keV) during 10 days.

\section{ANALYSIS OF DATA}

The behaviour of the ISO106 amplifiers looks very good: the offset output can be considered constant as the dose is increased. The $\mathrm{dc}$ and ac gains did not change. The input current is almost stable up to the fluence of $2.9 \times 10^{12} \mathrm{n} / \mathrm{cm}^{2}(\mathrm{E}>140 \mathrm{keV})$ and then decreases by $10-20 \%$ at the fluence of $1.1 \times 10^{13} \mathrm{n} / \mathrm{cm}^{2}$ (E>140 $\mathrm{keV}$ ). Although the ISO106 isolation amplifier behaves better than the AD289J, it needs additional amplification and an external power supply. Therefore it was not considered for further experiments.

The dc/dc converters, PWS727, show a low resistance to radiation. The input current of the components strongly increases as the dose increases $(>60 \%$ for a fluence of $2.9 \times 10^{12} \mathrm{n} / \mathrm{cm}^{2}(E>140 \mathrm{keV})$; the output voltages became noisy, unstable and for fluence larger than $2.9 \times 10^{12} \mathrm{n} / \mathrm{cm}^{2}(E>140 \mathrm{keV})$, the components failed.

As expected the magnetic amplifier is radiation resistant. The circuit was optimised by adjusting the ac voltage (Ea) at $200 \mathrm{~Hz}$ to minimise the voltage across the resistor $R$. The optimum value was found for an ac voltage of $10 \mathrm{~V}_{\text {mss }}$. Then, a signal of $0.5 \mathrm{~V}$ was applied to the control circuit and the resulting voltage was observed on $\mathrm{R}$ for different radiation doses (Fig. 3). Only a small dc drift was observed on the resistance $R$ with an increase of the dose level. According to these results, the magnetic amplifier is the best choice for its insensitivity to radiation, making it a good level detector in a high radiation zone. However it has low input impedance and non linear gain.

The AD289J show good resistance to the radiation up to a fluence of $3.3 \times 10^{13} \mathrm{n} / \mathrm{cm}^{2}$ $(E>140 \mathrm{keV})$ : small variations of the voltage offset (less than 10\%), stable dc gain, and a stable gain at
$100 \mathrm{~Hz}$ (gain of the circuit $=10$ ). The power consumption decreased by about $40 \%$ with an increase of the radiation dose: but this variation does not seem to affect the performance of the circuit. On the other hand, the performance of the AD289J was more radiation sensitive at higher gain (Fig. 4): the characteristics deteriorated quickly already for a gain of 30 . Their use in LHC is therefore limited to gains of 10 .

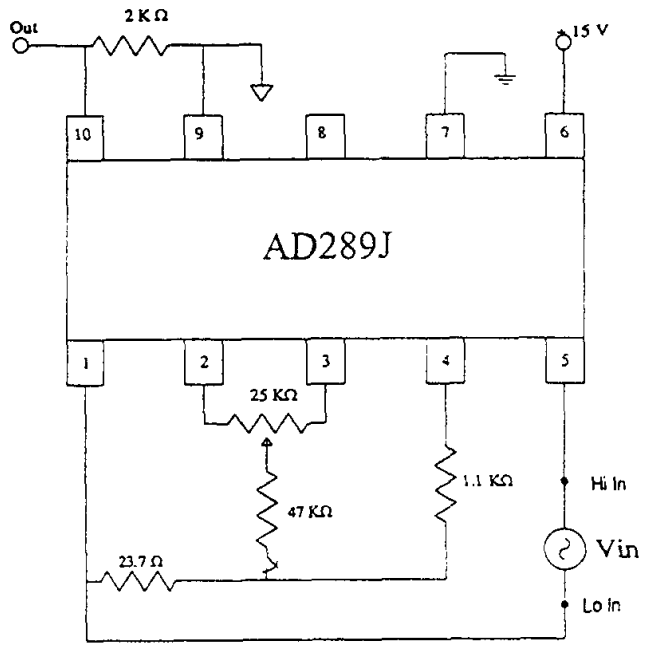

Fig.4. A) AD289: test circuit

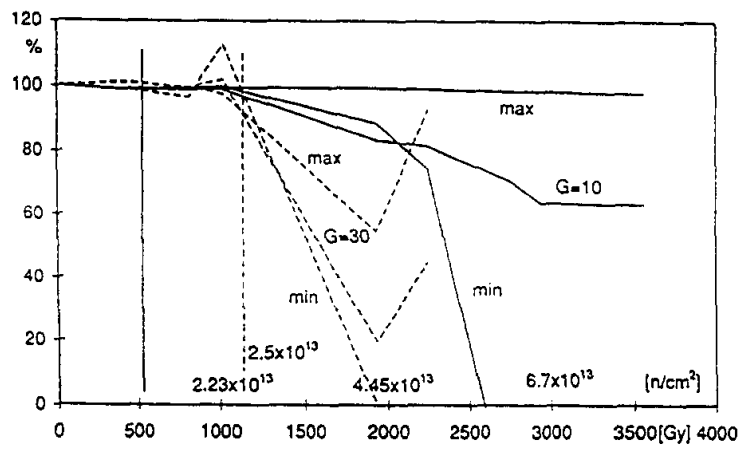

Fig. 4. B) AD289: dc gain vs. dose

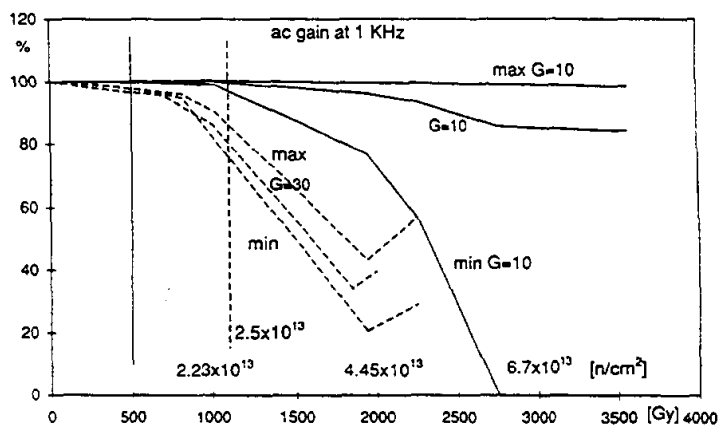

Fig. 4. C) AD289: ac gain at $1 \mathrm{kHz}$ 
The AD210JN reacted the best under radiation. The electrical characteristics stay stable up to a fluence of $5.6 \times 10^{13} \mathrm{n} / \mathrm{cm}^{2}(\mathrm{E}>140 \mathrm{keV})$ : only the power consumption decreased $(-40 \%)$ with the radiation level, dc and ac gain and offset show no appreciable variation (Fig. 5).

In figures 4 and 5 , the measurements of the electrical characteristics of every component have been

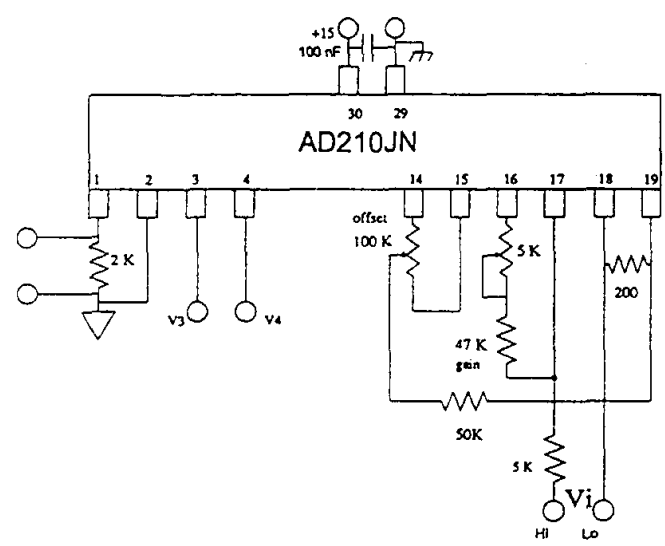

Fig. 5. A) AD210: test circuit

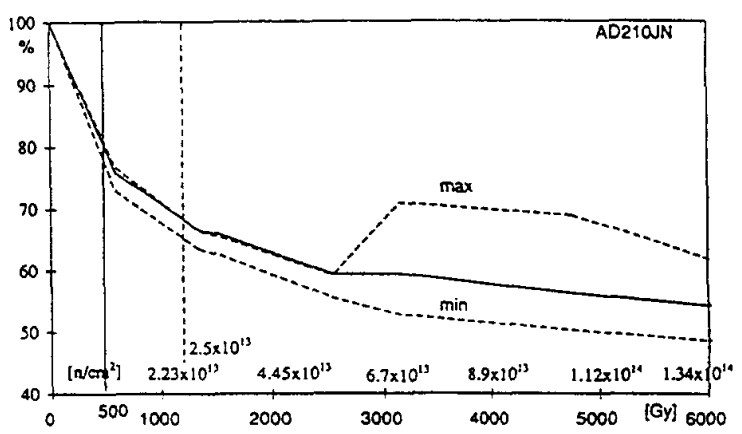

Fig. 5. B)AD210: Power consumption vs. dose

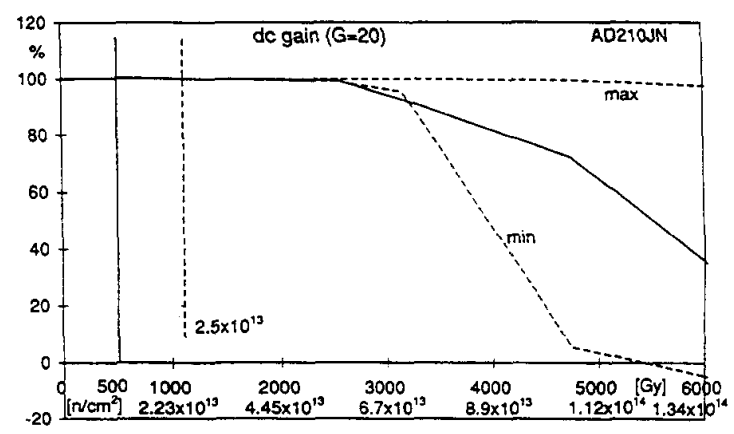

Fig. 5. C) AD210: dc gain $(G=20)$ vs dose

normalised to $100 \%$ for the original value prior to irradiation. The mean value of the samples has been drawn with the minimum/maximum deviations from the mean value for the different radiation doses.

\section{CONCLUSIONS}

The isolation amplifiers AD210JN gave very promising results. They all operated adequately up to a fluence of $5.6 \times 10^{13} \mathrm{n} / \mathrm{cm}^{2}(E>140 \mathrm{keV}$, which is equivalent to between 20 and 200 years of LHC running time, assuming a continuous loss of $10^{6}$ protons per second on the magnets. The input offset remained less than $2 \mathrm{mV}$ compared to quench detection levels between 0.2 to $0.5 \mathrm{~V}$ (still to be finally determined).

Moreover, the degradation of the circuits is gradual with the increase of the radiation dose. As a consequence, amplifiers can be monitored and eventually replaced if necessary during shut-down periods.

The current consumption decreases with the exposure, but the spread of values stay very narrow: $\pm 4 \mathrm{~mA}$ prior to irradiation, $\pm 2 \mathrm{~mA}$ at $5.6 \times 10^{13} \mathrm{n} / \mathrm{cm}^{2}$ (E>140 keV). This property could be used to monitor the dose.

\section{FUTURE DEVELOPMENTS}

Ten new AD210JN will be tested in the tunnel of the Super Proton Synchrotron (SPS) at CERN at a total dose level of 500 Gy over 1 year. Such condition (a dose rate only 10 times stronger then in LHC) should reflect more closely the real situation.

Power consumption will be continuously monitored all over this test, in order to verify that the decrease in power consumption is strictly related to the dose received.

\section{ACKNOWLEDGEMENT}

I wish to thank L. Coull for the many useful discussions concerning the LHC magnet quench protection system and his kind suggestions about the choice of the circuits to be tested. I am equally grateful to $M$. Tavlet for the support provided in organising the irradiation exposures and dose measurements.

\section{REFERENCES}

[1] L. Coull, D. Hagedorn, V. Remondino, F. Rodriguez-Mateos, "LHC Magnet Quench Protection System", MTI3,. Victoria, Canada, 1993.

[2] L. Coull, D. Hagedorn, V. Remondino, F. Rodriguez-Mateos "LHC Magnet Quench Protection System", CERN/AT MA/93-81.

[3] G.R. Stevenson, J.M. Zazula, "Estimates of Dose in the LHC Tunnel due to Beam-Gas Scattering", CERN/TIS-RP/93-6.

[4] L. Bumod, J.B. Jeanneret, H. Schonbacher, "Expected Doses inside and around LHC Dipoles", CERN/AC/DU/FA/Note 93. 06.

[5]M. Tavlet, E. L. Florian, "PSAIF: The PS-ACOL Irradiation Facility at CERN", Radecs 91, La Grande-Motte, France, 1991

[6] A. Rindi: Unfolding neutron spectra: LOUHI for pedestrians. LBL-6413 (1977)

[7] M. Tavlet, E. L. Florian, "Dose and Neutron-Fluence Measurements in Mixed Gamma-Neutron Fields by means of Semi-conductors Dosimeters", Radecs 93, IEEE Cat No 93TH0616-3

[8] K.H. Mess (Desy), Private communication. 
